\title{
Adaptive observer approach for actuators multiplicative faults detection and isolation
}

\author{
Raouaa TAYARI and Fayçal BEN HMIDA \\ Research unit C3S, ESSTT, University of Tunis, 5 av. Taha Hussein BP $56-1008$ Tunis, Tunisia \\ E-mails: tayari.raouaa@ hotmail.com, faycel.benhmida@esstt.rnu.tn
}

\begin{abstract}
In this paper we interest in the problem of multiplicative faults detection and isolation for linear systems. To solve this problem we use a bank of adaptive observers. The proposed algorithm is applied on a numerical example to conclude that the correct and robust detection and isolation in respect to the actuators multiplicative faults.
\end{abstract}

Keywords: multiplicative faults, faults detection and isolation, adaptive observer.

\section{INTRODUCTION}

Industrial systems are becoming increasingly complicated and sophisticated, a strong interest is therefore manifested concerning the monitoring and safety of these systems. The reasons why the industry is interested in the monitoring and safety of systems are the safety (human and environmental) and the necessity of increasing the productivity and the reliability. This is shown in the emersion of surveillance methods whose purpose is the fault detection and isolation (FDI), for this reason it become a domain of increasing importance in research and application. Many approaches FDI based-model and among these approaches those build on the state observers with [1].

The methods based on observers are adopted by [2] and [3], in which the generation of residues are used to calculate the difference between the actual outputs and those estimated like in [4], [5]. These methods are very effective for the fault detection and isolation.

Process abnormalities are usually classified into additive or multiplicative faults according to the effects on process. The multiplicative faults usually have important effects on the process dynamics, and is much less well studied compared to the additive faults such as in [6]. For this reason we will focus on the multiplicative faults using the adaptive observer which are supplied for example by [7], [8].

The adaptive observer estimate simultaneously the state and the system parameters and makes the design of the observer gain matrix easier. Sufficient conditions for the proposed observer is provided in the FDI. It shows that the proposed approach is robust.

In this paper, we focus on faults detection and isolation of multiplicative faults. To facilitate the FDI we propose to transform the multiplicative faults to additive faults based on adaptive observer. To explain this method, we present in Section 2 a problem formulation and the fault modeling. In Section 3, a structure of adaptive observer is proposed.
Then in section 4, we use the type of observer for actuators multiplicative faults detection and isolation. A numerical application to show the usefulness and robustness of this approach is illustrated in section 5. Finally, concluding remarks are given in Section 6.

\section{PROBLEM FORMULATION}

Consider the following linear system

$$
\begin{gathered}
\dot{x}(t)=A x(t)+B u(t)+B\left(I-\Sigma_{A}\right)\left(u_{l}^{f}(t)-u(t)\right) \\
y(t)=C x(t)
\end{gathered}
$$

where $x \in \Re^{n}$ is the state, $u \in \Re^{m}$ is the input, $y \in \Re^{p}$ is the measured output; $A \in \Re^{n \times n}, B \in \Re^{n \times m}, C \in \Re^{p \times n}$ are constant matrices; $u(t)$ is the command signal without fault and $u_{l}^{f}(t) \in \Re^{m}$ is an unknown vector. The term $\Sigma_{A}$ is defined by $\Sigma_{A}=\operatorname{diag}\left\{\sigma_{1}^{a}, \sigma_{2}^{a} \ldots . . \sigma_{m}^{a}\right\}$ with $\sigma_{i}^{a} \in\left[\begin{array}{ll}0 & 1\end{array}\right]$ for $i=1,2 \ldots . m$. So $\sigma_{i}^{a}=0$ presents a fault causing failure of $i_{t h}$ actuator of the system, so that the control action coming from this $i_{t h}$ actuator becomes equal to the $i_{t h}$ element of the uncontrollable offset vector $u_{l}^{f}(t)$ that is to say $u_{f}(i)=$ $u_{l}^{f}(i)$. On the other hand, $\sigma_{i}^{a}=1$ implies that the $i_{t h}$ actuator operates normally, i.e. $u_{f}(i)=u(i)$. Finally we can say that $\left.\sigma_{i} \in\right] 01[$ in case of partial fault.

Rodrigues [9] proposed a method to transform multiplicative faults to additive faults. Hence, the state equation (1) affected by actuators multiplicative faults becomes:

$$
\dot{x}(t)=A x(t)+B u(t)+B f(t)
$$

with

$f(t)=\left(I-\Sigma_{A}\right)\left(u_{l}^{f}(t)-u(t)\right)$ is the additive faults

Which the state equation (3) is equivalent to:

$$
\dot{x}=A x+\sum_{j \neq l}^{m} b_{j} u_{j}+b_{l} u_{l}^{f}
$$

where $B=\left[b_{1} \ldots . b_{m}\right]$

Our objective is to give a robust system for detection and isolation of actuators multiplicative faults. For this, we use the modeling of Rodrigues (3) by applying on the adaptive observer which is based on the residual generation. 


\section{BANK OF ADAPTIVE OBSERVERS DESIGN}

The following diagram shows the structure of the generalized observer for actuators multiplicative faults isolation:

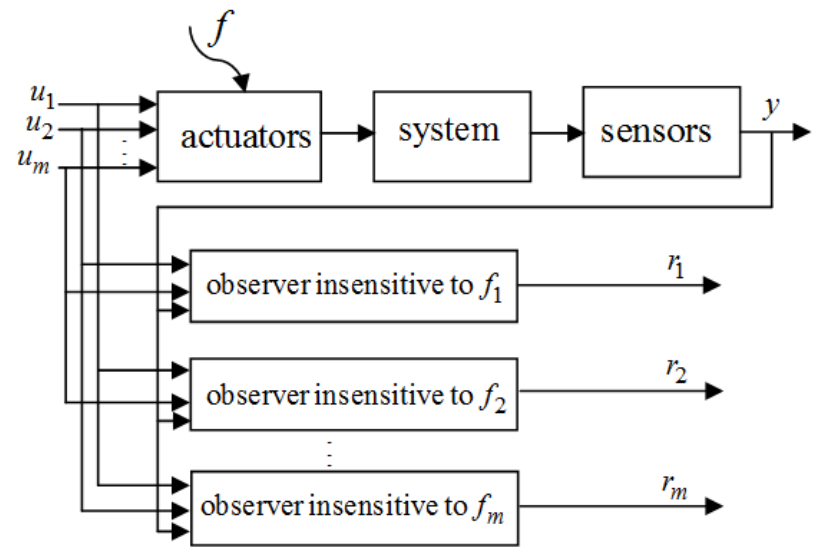

Fig. 1. A robust actuator fault isolation scheme.

Consider the following state equation of the system in nominal conditions

$$
\dot{x}(t)=A x(t)+B u(t)
$$

Envisaging the system (5) has $m$ inputs corresponding to $m$ sets of actuators, there $m$ possible faults models for the actuator. Assuming that the $l_{t h}$ actuator is faulty, then for $t \geq t_{f}$ the output of this actuator will be $u_{l}^{f}=u_{l}+f_{a l}$ and $\lim _{t \rightarrow \infty}\left|u_{l}^{f}-u_{l}\right| \neq 0$, where $t_{f}$ is the instant of occurrence of fault, $u_{l}$ is the $l_{t h}$ healthy actuator output, $u_{l}^{f}$ is a faulty constant output of $l_{t h}$ actuator.

Therefore, the corresponding faults model is that of state equation (4).

A bank of adaptive observers can be designed for the faults models as follows [10]:

$$
\left\{\begin{array}{l}
\dot{\hat{x}}_{i}=A \hat{x}_{i}-L\left(\hat{y}_{i}-y\right)+\sum_{j \neq i}^{m} b_{j} u_{j}+b_{i} \hat{u}_{i}^{f} \\
\dot{\hat{u}}_{i}^{f}=-2 \gamma_{i}\left(\hat{y}_{i}-y\right)^{T} F_{i} \\
\hat{y}_{i}=C \hat{x}_{i}
\end{array}\right.
$$

for $i=1,2 \ldots \ldots m, \hat{x}_{i}$ is the estimate of the state $x$ in the presence of fault in the $i_{t h}$ actuator, $\gamma$ is a design constant positive.

To design a faults isolation observer, the following Lemma is needed.

\section{Lemma:}

If there exists two symetric, positive definite matrices $P$ and
$Q$ and matrices $L$ and $F=\left[F_{1} \ldots \ldots . . F_{m}\right]$ that satisfies the following conditions:

$$
\begin{gathered}
(A-L C)^{T} P+P(A-L C)=-Q \\
P b_{i}=C^{T} F_{i}
\end{gathered}
$$

Then, the state estimation error is asymptotically stable.

\section{ACTUATOR FAULT DETECTION AND ISOLATION}

We consider the problem of faults detection and isolation based on adaptive observers, whose objective is to create a bank of adaptive observer. We use the method of the generation of residues for the resolution of this problem.

The residue is defined as the norm 2 of the difference between the actual value and the estimated value of the output to make a good faults detection and isolation [11]:

$$
r_{i}(t)=\left\|\hat{y}_{i}(t)-y(t)\right\|^{2} \quad \text { with } i \in 1,2 \ldots . m
$$

If the $l_{t h}$ actuator is faulty we distinguish two cases: when $i=l$

$$
\lim _{t \rightarrow \infty}\left(\hat{x}_{l}-x\right)=0
$$

with

$$
\left(\dot{\hat{x}}_{l}-\dot{x}\right)=(A-L C) e_{x_{l}}+b_{l} \tilde{u}_{l}^{f}
$$

and when $i \neq l$

$$
\lim _{t \rightarrow \infty}\left(\hat{x}_{i}-x\right) \neq 0
$$

with

$$
\left(\dot{\hat{x}}_{i}-\dot{x}\right)=(A-L C)\left(x_{i}-x\right)+b_{l}\left(u_{l}-u_{l}^{f}\right)-b_{i}\left(u_{i}-\hat{u}_{i}^{f}\right)
$$

Monitoring all residuals $\left[r_{1}(t) \ldots, r_{l}(t) \ldots \ldots . r_{m}(t)\right]$, it can be conclude that, if there exist $l \in 1,2 \ldots m$, when $\lim _{t \rightarrow \infty} r_{l}(t)=0$ while $\lim _{t \rightarrow \infty} r_{i}(t) \neq 0$ for all $i \neq l$, the $l_{t h}$ actuator is faulty. 
The sufficient condition for actuators isolation is satisfied, if the matrix $C B$ has full column rank. The faults actuators isolation can be achieved by measuring the residuals from the bank of observers described by (6).

\section{ILLUSTRATIVE EXAMPLE}

Consider the following system used in Watanabe and Himmelblau [12], with the following parameters matrices:

$$
\begin{aligned}
& A=\left[\begin{array}{cccc}
-3.6 & 0 & 0 & 0 \\
0 & -3.6702 & 0 & 0.0702 \\
0 & 0 & -36.2588 & 0.2588 \\
0 & 0.6344 & 0.7781 & -1.4125
\end{array}\right] \\
& B=\left[\begin{array}{ccc}
1 & 0 & 0 \\
0 & 1 & -1 \\
1 & 0 & 1 \\
0 & 0 & 0
\end{array}\right] \quad C=\left[\begin{array}{llll}
1 & 0 & 0 & 0 \\
0 & 1 & 0 & 0 \\
0 & 0 & 1 & 0
\end{array}\right]
\end{aligned}
$$

We verify that the matrix $C B$ is full rank column, and hence a bank of faults isolation actuators based adaptive observers is builded. We fix the poles of the estimator to $\{-72.5292,-2.7910,-7.3628,-7.2000\}$.

With the above parameters, we obtain

$$
\begin{aligned}
& P=\left[\begin{array}{llll}
1 & 0 & 0 & 0 \\
0 & 1 & 0 & 0 \\
0 & 0 & 1 & 0 \\
0 & 0 & 0 & 1
\end{array}\right] \\
& L=\left[\begin{array}{ccc}
31.5258 & 5.3334 & 24.2693 \\
9.8936 & 5.5047 & 8.9560 \\
40.8260 & 8.1236 & 7.9110 \\
240.0688 & 53.0741 & 236.3485
\end{array}\right] \\
& Q=\left[\begin{array}{cccc}
70.2516 & 15.2270 & 65.0954 & 240.0688 \\
15.2270 & 18.3498 & 17.0796 & 52.6405 \\
65.0954 & 17.0796 & 88.3396 & 235.3116 \\
240.0688 & 52.6405 & 235.3116 & 2.8250
\end{array}\right] \\
& \text { with } F_{1}=\left[\begin{array}{l}
1 \\
0 \\
1
\end{array}\right], F_{2}=\left[\begin{array}{l}
0 \\
1 \\
0
\end{array}\right], F_{3}=\left[\begin{array}{c}
0 \\
-1 \\
1
\end{array}\right] \\
& \text { and } \gamma_{1}=\gamma_{2}=\gamma_{3}=15
\end{aligned}
$$

\section{A. The generation of residues without measurement noise and parameters variations}

The figure 2 shows the generation of residues with actuators fault free.
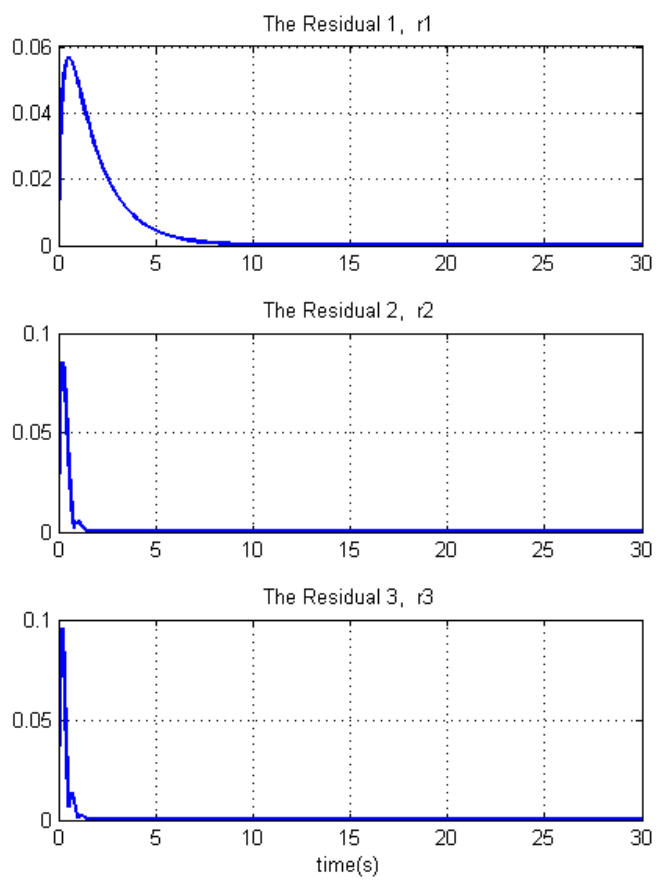

Fig. 2. Residues in the absence of fault on three actuators.

The figures 3-5 illustrate the generation of residues with actuators faults. We introduce an abrupt fault with amplitude equal to 0.5 at time $t=15 \mathrm{~s}$.
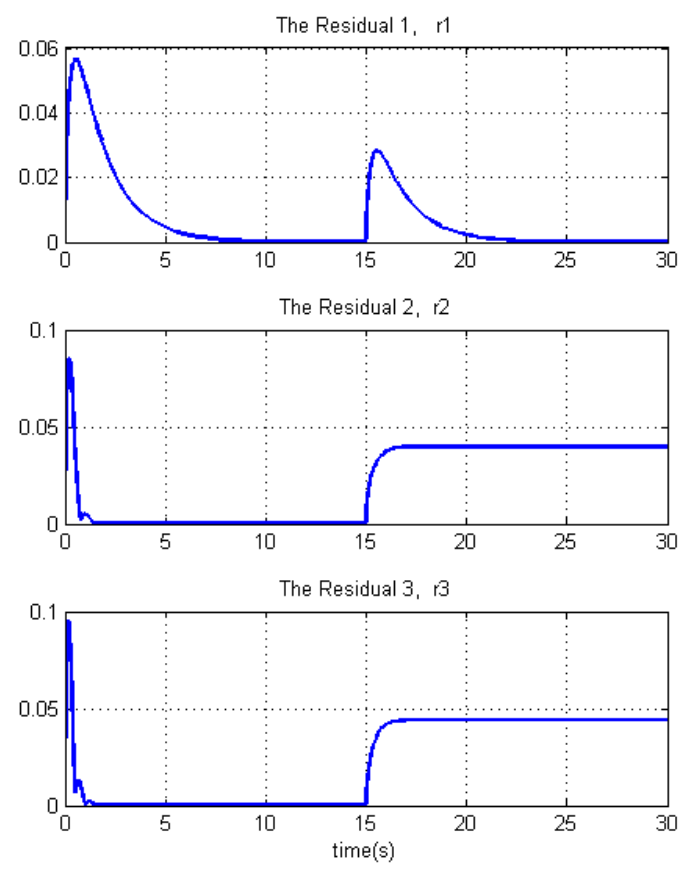

Fig. 3. Residues in presence of fault on the first actuator. 

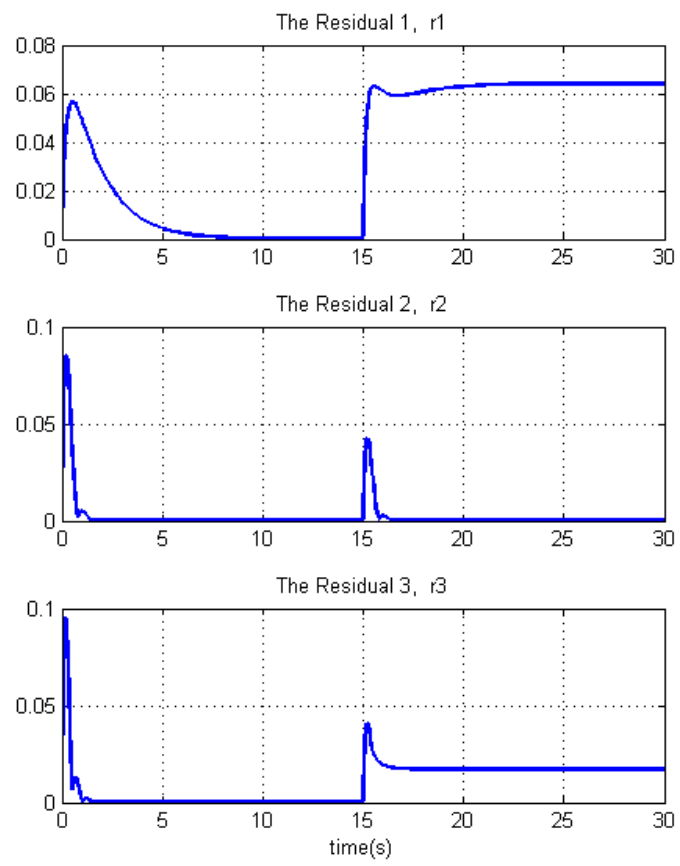

Fig. 4. Residues in presence of fault on the second actuator.
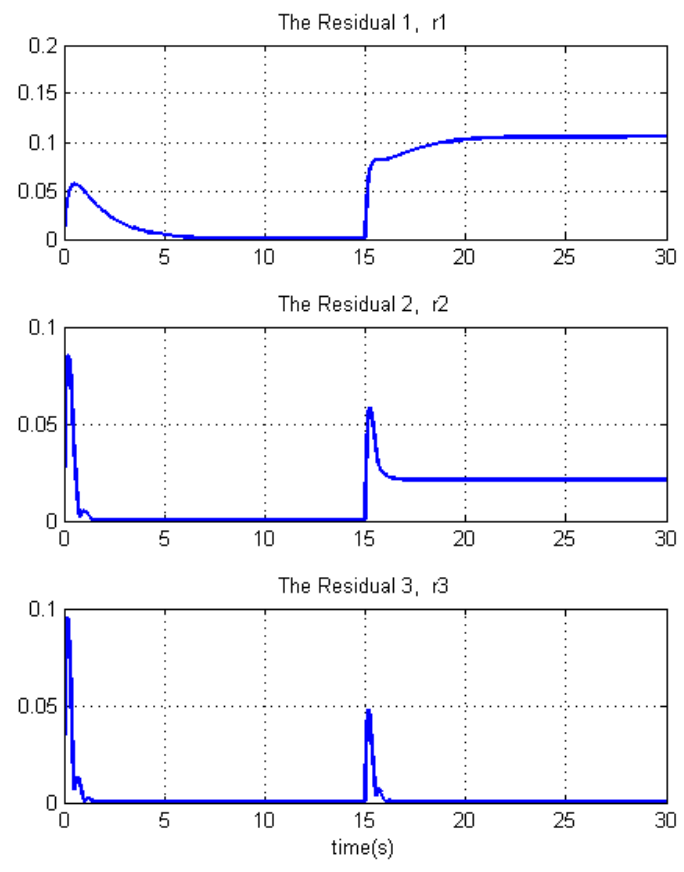

Fig. 5. Residues in presence of fault on the third actuator.
It can be concluded from figure (2), the three residues converge to zero in absence of faults, in the meanwhile the figures 3-5 can show that the faulty residuals goes to zero while the healthy does not.

TABLE I

FAULTS SIGNATURES

\begin{tabular}{|l|l|l|l|}
\hline & $r_{1}$ & $r_{2}$ & $r_{3}$ \\
\hline$f_{1}$ & 0 & 1 & 1 \\
\hline$f_{2}$ & 1 & 0 & 1 \\
\hline$f_{3}$ & 1 & 1 & 0 \\
\hline
\end{tabular}

where $f_{1}, f_{2}, f_{3}$ are the faults, $r_{1}, r_{2}, r_{3}$ are the residue, $1=$ residual is sensitive to fault and $0=$ residual is insensitive to fault.

This table presents that we can isolate many types of faults with this approach.

\section{B. Residual generation with measurement noise}

We introduce a white noise on each of the measuring signals with a variance of $10^{(-5)}$ and with zero average.
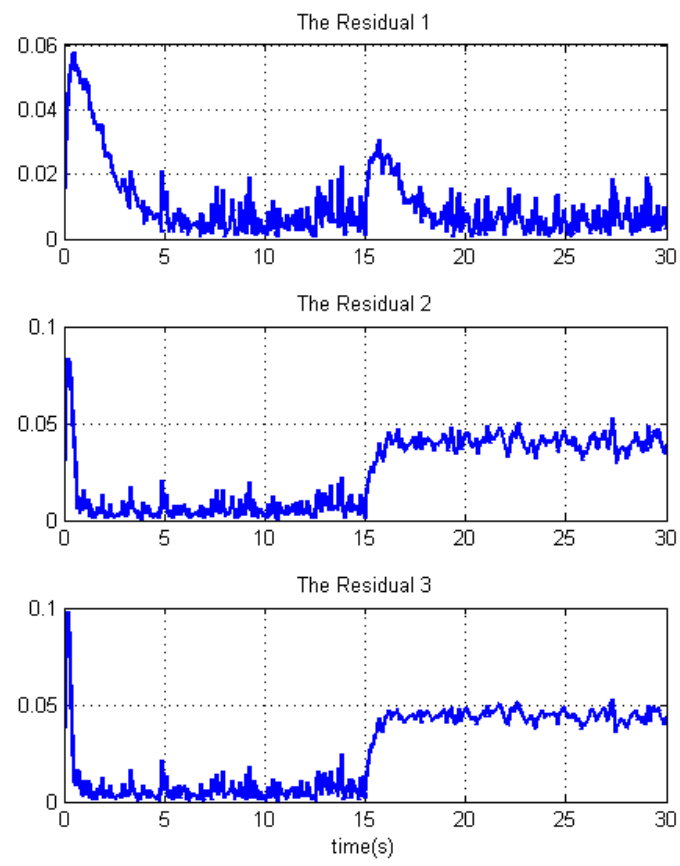

Fig. 6. Residues in presence of fault on the first actuator and in presence of noise on the measurement. 

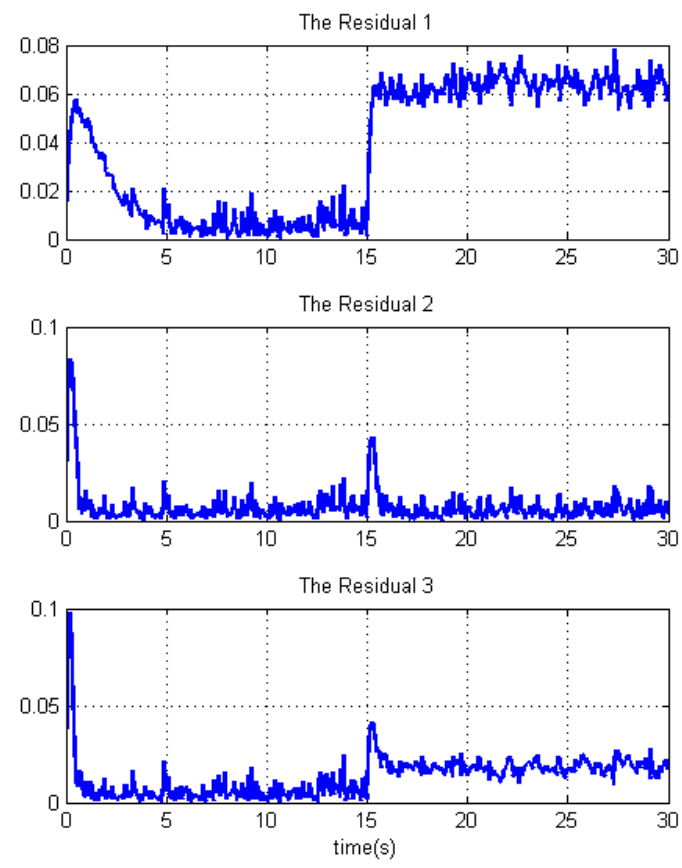

Fig. 7. Residues in presence of fault on the second actuator and in presence of noise on the measurement.
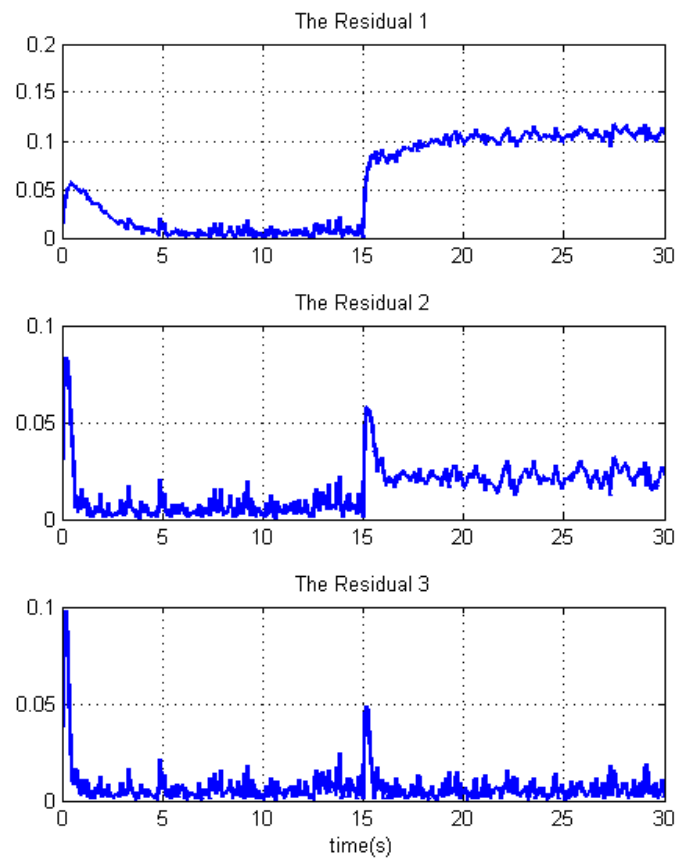

Fig. 8. Residues in presence of fault on the third actuator and in presence of noise on the measurement.

The figures 6-8 show that the residuals are robust with respect to noise and hence the fault isolation is done without problem.

\section{Residual generation with parameters variations}

In this section we add parametric variations in the fourth row of the state matrix, ie:

$$
\begin{aligned}
& A=A_{\text {nom }}+\Delta A \\
= & {\left[\begin{array}{cccc}
-3.6 & 0 & 0 & 0 \\
0 & -3.6702 & 0 & 0.0702 \\
0 & 0 & -36.2588 & 0.2588 \\
0 & 0.6344 & 0.7781 & -1.4125
\end{array}\right] } \\
+ & {\left[\begin{array}{cccc}
0 & 0 & 0 & 0 \\
0 & 0 & 0 & 0 \\
0 & 0 & 0 & 0 \\
\Delta 41 & \Delta 42 & \Delta 43 & \Delta 44
\end{array}\right] }
\end{aligned}
$$

where $\Delta 41, \Delta 42, \Delta 43, \Delta 44$ are the parametric variations in the fourth row of the state matrix.

The figures 9 and 10 illustrate that the adaptive observer is robust despite the parameters variations so the faults isolation is done.

Parametric variations of $10 \%$ :
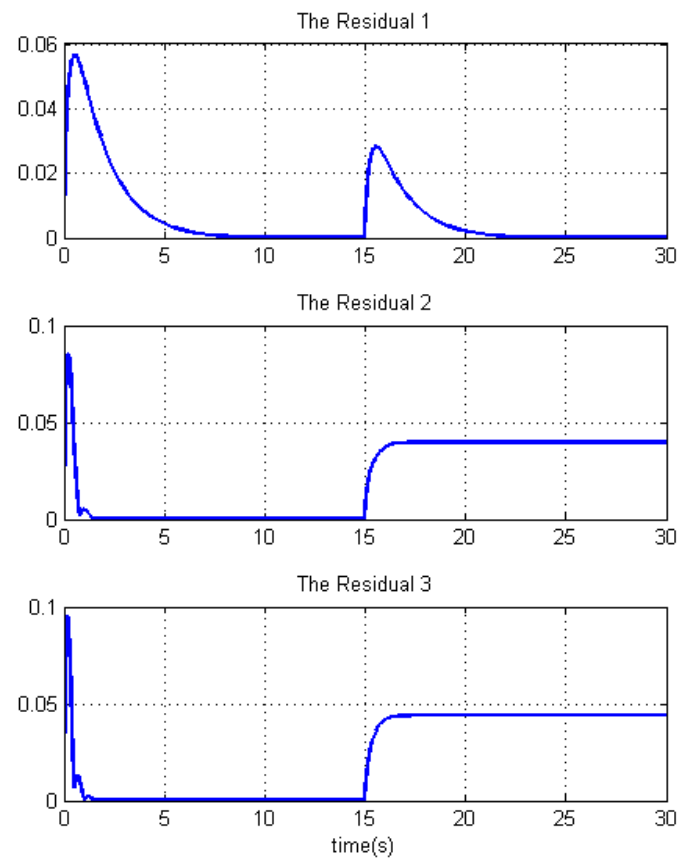

Fig. 9. Residues in presence of fault on the first actuator and in presence of parameters variations. 
Parametric variations of $40 \%$ :
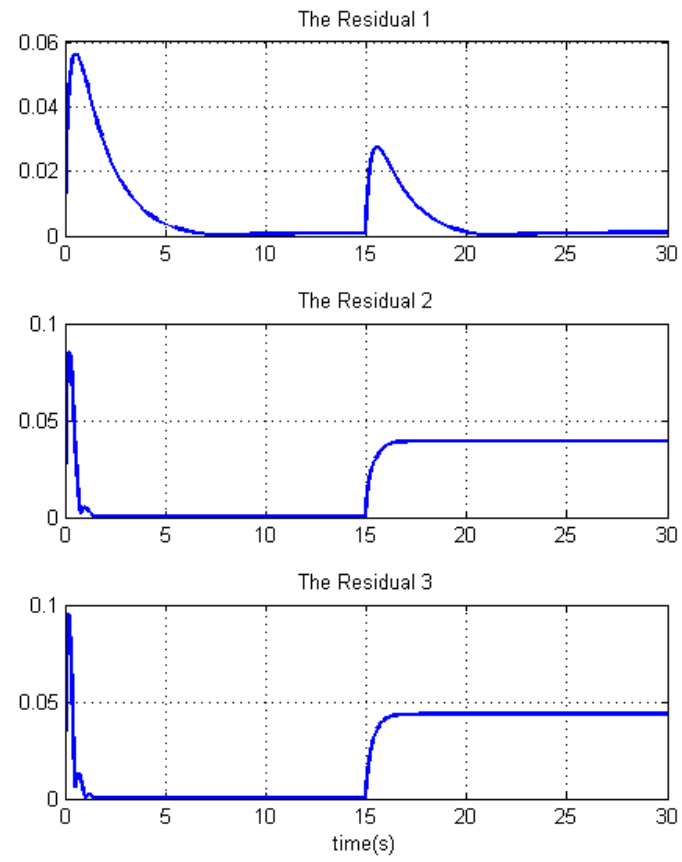

Fig. 10. Residues in presence of fault on the first actuator and in presence of parameters variations.

\section{CONCLUSION}

In this paper, a method of detection and isolation of actuators multiplicative faults for linear system is presented. This method is based on bank of adaptive observers. The idea is to transform multiplicative faults in additive. An illustrative example is presented to show the effectiveness of the proposed approach. The robustness of this observer with measurement noise and parameters variations have been also established.

\section{REFERENCES}

[1] R. N. Clark, D. C. Fosth and V. M. Walton, Detecting instrument malfunctions in control systems. IEEE Transactions on Aerospace and Electronic Systems, 11 (4), pp. 465-473, 1975.

[2] J. J. Gertler, Survey of model-based failure detection and isolation in complex plants. IEEE Control System Magazine, 8(6), pp. 3-11, 1988.

[3] M. Hou and P. C. Muller, Design of observers for linear systems with unknown inputs. IEEE Transaction on Automatic Control, AC- 37(6), pp. 871-875, 1992.

[4] J. Chen, R. Patton et H. Y. Zhang, Design of unknown input observers and robust fault detection filters. International Journal of control, 63(1), pp. 85-105, 1996.

[5] R. J. Patton, Fault-tolerant control: The 1997 situation. IFAC Safeprocess, Hull, UK, 2, pp. 1033-1054, 1997.

[6] H. P. Huang, C.C. Li and J. C. Jeng, Multiple Multiplicative Fault Diagnosis for Dynamic Processes via Parameter Similarity Measures. Industrial and Engineering Chemistry Research, 46(13), pp. 4517-4530, 2007.

[7] $\mathrm{H}$. Wang et S. Daley, Actuator fault diagnosis: an adaptive observerbased technique. IEEE transaction on Automatic Control, 41(7), pp. 1073-1078, 1996

[8] M. Siaha et M. A. Sadriana, Diagnosis using adaptive technique. Journal for applied sciences, 2002.
[9] M. Rodrigues, Diagnosis and tolerant active control defaults applied to systems described by multi- models linear. These Universities Henri Poincar, Nancy, France, 2005.

[10] J. Guangoing, Adaptive observer and sliding mode observers based actuators faults diagnosis for civil aircraft. These Universities Simon Fraser, 2003.

[11] W. Chen and M. Seif, An actuator fault isolation strategy for linear and nonlinear systems. American control conference, Portland, USA, 2005.

[12] K. Watanabe and D. M. Himmelblau, Instrument fault detection in systems with incertainties. Int. J. Sys. Sci, 13(2), pp. 137-158, 1982. 